\title{
Enhancing erythritol productivity in Yarrowia lipolytica using metabolic engineering
}

\author{
Frédéric Carly ${ }^{\mathrm{a}}$, Marie Vandermies ${ }^{\mathrm{b}}$, Samuel Telek ${ }^{\mathrm{b}}$, Sébastien Steels ${ }^{\mathrm{b}}$, Stéphane Thomas ${ }^{\mathrm{c}}$, \\ Jean-Marc Nicaud ${ }^{\mathrm{c}}$, Patrick Fickers ${ }^{\mathrm{b}, *}$

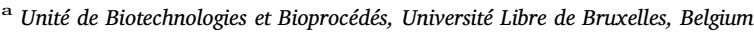 \\ ${ }^{\mathrm{b}}$ Microbial Processes and Interactions, TERRA Teaching and Research Centre, University of Liège - Gembloux Agro-Bio Tech, Belgium \\ ${ }^{\mathrm{c}}$ Micalis Institute, INRA, AgroParisTech, Université Paris-Saclay, 78350 Jouy-en-Josas, France
}

\section{A R T I C L E I N F O}

\section{Keywords:}

Yarrowia lipolytica

Erythritol

Metabolic engineering

Glycerol kinase

Transketolase

Erythrulose kinase

\begin{abstract}
A B S T R A C T
Erythritol (1,2,3,4-butanetetrol) is a four-carbon sugar alcohol with sweetening properties that is used by the agrofood industry as a food additive. In this study, we demonstrated that metabolic engineering can be used to improve the production of erythritol from glycerol in the yeast Yarrowia lipolytica. The best results were obtained using a mutant that overexpressed GUT1 and TKL1, which encode a glycerol kinase and a transketolase, respectively, and in which $E Y K 1$, which encodes erythrulose kinase, was disrupted; the latter enzyme is involved in an early step of erythritol catabolism. In this strain, erythritol productivity was $75 \%$ higher than in the wild type; furthermore, the culturing time needed to achieve maximum concentration was reduced by $40 \%$. An additional advantage is that the strain was unable to consume the erythritol it had created, further increasing the process's efficiency. The erythritol productivity values we obtained here are among the highest reported thus far.
\end{abstract}

\section{Introduction}

Erythritol (1,2,3,4-butanetetrol) is a four-carbon sugar alcohol with sweetening properties that is used by the agrofood industry as a food additive (E968). Erythritol is noncariogenic and has been determined to be safe for human consumption even when high doses are consumed daily (Bernt et al., 1996; Kawanabe et al., 1992). It has extremely low digestibility and does not modify blood insulin levels (Munro et al., 1998). Erythritol is widespread in nature and has been found in seaweed, fungi, fruit, and fermented food, although always at low levels (Moon et al., 2010). It is also produced by osmophilic microorganisms in response to osmotic stress (Hallsworth and Magan, 1997).

Although erythritol can be produced chemically from dialdehyde starch, this process has never been industrialized due to its low efficiency. Instead, erythritol is most commonly generated from glucose via fermentation processes using osmophilic yeasts (Moon et al., 2010) namely Aurobasidium sp. (Ishizuka et al., 1989), Trigonopsis variabilis (Kim et al., 1997), Torula sp. (Lee et al., 2000), Candida magnoliae (Ryu et al., 2000), Pseudozyma tsubakaensis (Jeya et al., 2009), and Moniliela sp. (Lin et al., 2010). Despite the some of these processes have been developed at industrial scale, they suffer from the high cost of the fermentation media and the production of unwanted byproducts such as mannitol, and organic acids which renders the downstream processing more challenging. Recently, Yarrowia lipolytica has also been found to be an efficient erythritol producer (Rymowicz et al., 2008; Yang et al., 2014; Park et al., 2016; Mirończuk et al., 2016).

$Y$. lipolytica is a non-conventional model yeast species that is wellknown for its unusual metabolic properties (Fickers et al., 2005; Nicaud, 2012). Because it can secrete large amounts of proteins and metabolites of biotechnological interest, Y. lipolytica has several industrial applications, including heterologous protein synthesis and citric acid production, and has been accorded GRAS status (Fickers et al., 2005; Zinjarde, 2014). Y. lipolytica is highly proficient at producing erythritol and can use raw glycerol instead of glucose as its main carbon source (Rymowicz et al., 2008; Almeida et al., 2012). Raw glycerol is a byproduct of the biodiesel production or fat industries (i.e. fat saponification, stearin synthesis) and is thus available in large quantities at lower price than glucose. Moreover, glycerol allows higher production yields as compared to glucose, making the erythritol production process more profitable. (Rymowicz et al., 2008; Tomaszewska et al., 2012; Rywińska et al., 2013). The synthesis of erythritol from glycerol is not a redox-balanced reaction, as it requires a net amount of oxidized cofactors. However it is more advantageous than using glucose since synthesis of erythritol from the latter consumes

\footnotetext{
*Correspondence to: Microbial Processes and Interactions, TERRA Teaching and Research Centre, University of Liège - Gembloux AgroBio Tech, Passage des Déportés, 2, 5030 Gembloux, Belgium.

E-mail address: pfickers@ulg.ac.be (P. Fickers).
} 


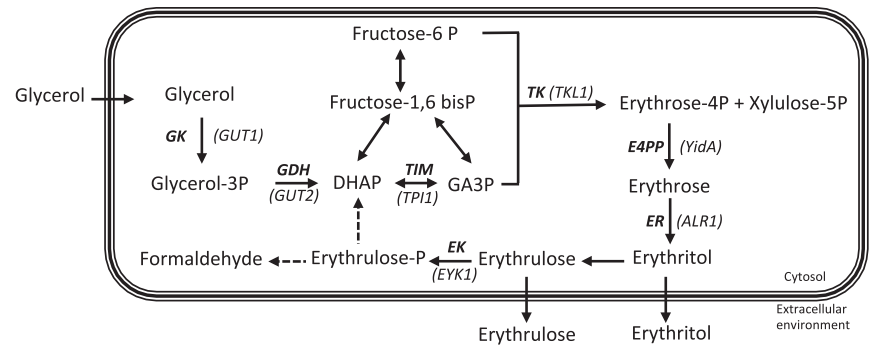

Fig. 1. Pathway used by Y. lipolytica to synthesize erythritol from glycerol. DHAP: dihydroxyacetone phosphate; GA3P: glyceraldehyde-3-phosphate; GK: glycerol kinase; GDH: glycerol-3P dehydrogenase; TIM: triosephosphate isomerase; TK: transketolase; E4PP: erythrose-4P phosphatase; ER: erythrose reductase; and EK: erythrulose kinase. Dotted arrows represent the hypothetic catabolism pathway of erythrulose-P according to Paradowska and Nitka (2009).

reduced cofactors that must be replenished through glucose oxidation.

When erythritol is synthesized from glycerol, the latter is first phosphorylated by a glycerol kinase (GK) before subsequently being dehydrogenated by a glycerol-3P-dehydrogenase (GDH), giving rise to dihydroxyacetone phosphate (DHAP) (Fig. 1). DHAP is then converted by a triosephosphate isomerase (TIM) into glyceraldehyde-3-phosphate, which enters into the pentose phosphate pathway, where a transketolase (TK) converts it into erythrose-4-phosphate. The latter is dephosphorylated by an erythrose-4P phosphatase (E4PP) and reduced by an erythrose reductase (ER) to become erythritol. Depending on experimental conditions, $Y$. lipolytica can also use erythritol as its main carbon source. We have recently highlighted that this catabolic pathway in $Y$. lipolytica is similar to those present in other yeasts, including Lipomyces starkeyi (Carly et al., submitted for publication) (Fig. 1). Furthermore, we identified the gene EYK1 (YALIOF01606g), which encodes an erythrulose kinase (EK). In Y. lipolytica, disruption of EYK1 impairs growth on erythritol.

Although most of the genes involved in erythritol synthesis have been described, little is known about their regulation at finer scales or about the pathway's limiting steps. To date, most studies seeking to improve erythritol production have used wild type strains or randomly generated mutants and have focused on optimizing the culture medium or culturing conditions (Rymowicz et al., 2008; Yang et al., 2014; Mirończuk et al., 2015; Rakicka et al., 2016). However, some recent research using $Y$. lipolytica has underscored the potential utility of genetic engineering approaches. Mirończuk et al. (2016) found that the constitutive expression of genes encoding GK and GDH (i.e., GUT1 and GUT2, respectively) in the $Y$. lipolytica A101 strain led to a significant increase in glycerol uptake capacity. Overexpression of these genes resulted in significant increases in erythritol productivity $23 \%$ for GUT1 only and $35 \%$ for both) in the mutants as compared to the wild type strain; in contrast, overexpression of GUT2 alone led to a $28 \%$ decrease in erythritol productivity. Other enzymes merit some attention as well. Transketolase (TK) has been described as a key enzyme in erythritol synthesis in Trichosporonoides megachiliensis (Sawada et al., 2009) similarly to erythrose reductase (ER) in Candida magnolia (Ghezelbash et al., 2014). Furthermore, using proteomics, Yang et al. (2015) highlighted the importance of triose-phosphate isomerase (TIM) in erythritol synthesis in Y. lipolytica.

In this study, we sought to identify additional limits acting on erythritol production and thus further enhance this process. To this end, we constructed a set of strains that overexpressed genes encoding for key enzymes in the erythritol synthesis pathway. We then studied the effects of different gene combinations on erythritol synthesis. In the most productive mutants, EYK1 was also disrupted to further increase erythritol productivity.

\section{Materials and methods}

\subsection{Strains, media, and culture conditions}

The Escherichia coli and Y. lipolytica strains used in this study are listed in Supplementary table 1 . The E. coli strains were grown at $37^{\circ} \mathrm{C}$ in Luria-Bertani medium supplemented with kanamycin sulfate (50 mg/L; Sigma-Aldrich). The $Y$. lipolytica strains were grown at $28{ }^{\circ} \mathrm{C}$ in YNB medium supplemented to meet the requirements of auxothrophs (Barth and Gaillardin, 1996); EG medium (glycerol $50 \mathrm{~g}$ / $\mathrm{L}$, yeast extract $5 \mathrm{~g} / \mathrm{L}$ and peptone $5 \mathrm{~g} / \mathrm{L}$ ); EPF medium (glycerol $100 \mathrm{~g} /$ $\mathrm{L}$, yeast extract $1 \mathrm{~g} / \mathrm{L}, \mathrm{NH}_{4} \mathrm{Cl} 4.5 \mathrm{~g} / \mathrm{L}, \mathrm{CuSO}_{4} 0.7 \mathrm{mg} / \mathrm{L}, \mathrm{MnSO}_{4} \cdot \mathrm{H}_{2} \mathrm{O}$ $32 \mathrm{mg} / \mathrm{L}$, and $0.72 \mathrm{M}$ phosphate buffer $\mathrm{pH} 4.3$ ) or EPB medium (glycerol $150 \mathrm{~g} / \mathrm{L}, \mathrm{NH}_{4} \mathrm{Cl} 2 \mathrm{~g} / \mathrm{L}, \mathrm{KH}_{2} \mathrm{PO}_{4} 0.2 \mathrm{~g} / \mathrm{L}, \mathrm{MgSO}_{4} \cdot 7 \mathrm{H}_{2} \mathrm{O} 1 \mathrm{~g} / \mathrm{L}$, $\mathrm{NaCl} 25 \mathrm{~g} / \mathrm{L}$ and yeast extract $1 \mathrm{~g} / \mathrm{L})$. For solid media, agar $(15 \mathrm{~g} / \mathrm{L})$ was added. Shake-flask cultures were performed in triplicate for eight days in a rotary shaker at $28{ }^{\circ} \mathrm{C}$ and $190 \mathrm{RPM}$. After $72 \mathrm{~h}$ of preculturing in $35 \mathrm{~mL}$ of EG medium, cells were transferred to a $250 \mathrm{~mL}$-flask containing $35 \mathrm{~mL}$ of EPF medium; initial optical density at $600 \mathrm{~nm}\left(\mathrm{OD}_{600}\right)$ was 0.4 . Bioreactor cultures were performed in duplicate in a 2-L Biostat BTwin fermentor (Sartorius) containing $1 \mathrm{~L}$ of EPB medium and kept at $28{ }^{\circ} \mathrm{C}$. Stirrer speed was set to $800 \mathrm{RPM}$, and the aeration rate was $1 \mathrm{~L} /$ min. The $\mathrm{pH}$ was automatically maintained at 3.0 by the addition of $20 \%(w / v) \mathrm{NaOH}$ or $40 \%(w / v) \mathrm{H}_{3} \mathrm{PO}_{4}$.

\subsection{Quantifying biomass, glycerol concentration, and erythritol concentration}

Biomass was determined gravimetrically after the cells had been washed and dried at $105{ }^{\circ} \mathrm{C}$ for $24 \mathrm{~h}$. Glycerol and erythritol concentrations were determined using an HPLC (Agilent 1200 series; Agilent Technologies) equipped with a refractive index detector and an Aminex HPX-87H ion exclusion column $(300 \times 7.8 \mathrm{~mm}$; Bio-Rad). Elution was performed using $15 \mathrm{mM}$ trifluoroacetic acid as the mobile phase at a flow rate of $0.5 \mathrm{~mL} / \mathrm{min}$ and a temperature of $65^{\circ} \mathrm{C}$.

\subsection{General molecular biology techniques}

Standard media and techniques were used for E. coli (Sambrook et al., 1989), and the media and techniques used for Y. lipolytica have been described elsewhere (Barth and Gaillardin, 1996). The restriction enzymes, DNA polymerases, and ligase were supplied by Thermo Fisher Scientific. Genomic DNA from Y. lipolytica was prepared in accordance with Querol et al. (1992). PCR was performed using the primers listed in Supplementary table 2. DreamTaq DNA polymerase (Thermo Scientific) was used for cloning, and ExTaq DNA polymerase (Takara) was used to verify genomic structure. The PCR fragments were purified from the agarose gels using a GeneJet Gel Extraction Kit (Thermo Scientific). DNA sequencing was performed by GATC Biotech (https://www.gatcbiotech.com), and the primers were synthetized by Eurogentec (https:// secure.eurogentec.com/).

\subsection{Strain construction}

Details on the genes that were overexpressed in $Y$. lipolytica are provided in Table 1. The Y. lipolytica genes (namely, GUT1, GUT2, TPI1, TKL1) were amplified from the genomic DNA of JMY2900. Gene YidA was amplified from $E$. coli strain BL21, and gene ALR was amplified from $C$. magnolia strain CBS2800. A synthetic, codon-optimized version of ALR (ALR_CO) specific to Y. lipolytica was obtained from GeneArt (https://www.thermofisher.com/geneart). Codon content of gene $A L R$ was compared to $Y$. lipolytica CLIB122 codon usage table using Graphical Codon Usage Analyzer CGUA (http://gcua.schoedl.de/) and rare codons (below $20 \%$ in frequency in $Y$. lipolytica) were replaced with the most common codons coding for the same amino acid (Supplementary Fig. 1). All the gene-amplification primers were 
Table 1

Genes overexpressed in Y. lipolytica.

\begin{tabular}{|c|c|c|c|c|}
\hline Reference & Name & Enzyme encoded & Origin & Modification \\
\hline YALIOF00484g & GUT1 & Glycerol kinase & Y. lipolytica & $\begin{array}{l}\text { BamHI site } \\
\text { removed }\end{array}$ \\
\hline YALIOB13970g & GUT2 & $\begin{array}{l}\text { Glycerol-3P } \\
\text { dehydrogenase }\end{array}$ & Y. lipolytica & BgIII site removed \\
\hline YALIOF05214g & TPI1 & Triose-P isomerase & Y. lipolytica & \\
\hline YALIOE06479g & $T K L 1$ & Transketolase & Y. lipolytica & $\begin{array}{l}\text { Intron removed, } \\
\text { ClaI site removed }\end{array}$ \\
\hline EG11195 & YidA & $\begin{array}{l}\text { Erythrose-4P } \\
\text { phosphatase }\end{array}$ & E. coli & \\
\hline FJ550210 & $A L R$ & $\begin{array}{l}\text { Erythrose } \\
\text { reductase }\end{array}$ & C. magnoliae & \\
\hline
\end{tabular}

designed to introduce an AvrII site at the $3^{\prime}$ end and either a BamHI or a BglII restriction site at the $5^{\prime}$ end (Supplementary table 2). Introns and undesirable restriction sites were removed by overlap extension PCR and site-directed mutagenesis (Higuchi et al., 1988; see Table 1 for details). Amplicons were purified from the agarose gel and then digested using BamHI/AvrII or BglII/AvrII restriction enzymes. The corresponding fragments were subsequently cloned into BamHI/AvrII digested JMP1047 or JMP2563 vectors to obtain URA3 or LEU2selectable plasmids, respectively (Supplementary table 1 ). The correctness of the resulting constructs was verified by DNA sequencing.

Strain RIY203 was constructed by disrupting the EYK1 gene in the Po1d strain as described elsewhere (Vandermies et al., 2017, in press). EYK1 P and T fragments were amplified from Po1d genomic DNA using primer pairs EYK1-PF/EYK1-PR and EYK1-TF/EYK1-TR, respectively. The URA3 marker was amplified from the JMP113 plasmid using the primer pair LPR-F/LPR-R. Amplicons were digested with SfiI before being purified and ligated, using T4 DNA ligase. The ligation products were amplified via PCR using the primer pair EYK1-PF/EYK1-TR. They were then purified and used to transform the Po1d strain. The result was strain RIY147, from which the URA3 marker was popped out using the Cre-lox recombination system and the replicative vector pRIP132 (Vandermies et al., 2017, in press); this process yielded strain RIY203.

Expression cassettes for the GUT1, GUT2, TPI1, TKLI, YidA, ALR, and ALR_CO genes were rescued from corresponding vectors by NotI digestion. They were then purified from the agarose gel and used to transform Y. lipolytica strains Po1d or RIY203 (Supplementary Fig. 2). Transformants were selected using YNB medium supplemented with uracil or leucine, depending on the nature of their auxotrophy. The correctness of the strain construction was verified by performing analytical PCR on genomic DNA; depending on the marker, primer pair URA3F/61stop or LEU2F/61stop was employed. Prototrophic stains were obtained by transforming the mutants with either the JMP1047 or JMP2563 plasmid, depending on the nature of the strain's auxothrophy.

\subsection{RNA isolation and transcript quantification}

Shake-flask cultures were grown in EPF medium for $24 \mathrm{~h}$. Cells were then collected at an $\mathrm{OD}_{600}$ of 0.5 and stored at $-80^{\circ} \mathrm{C}$. RNA extraction and cDNA synthesis were performed as previously described (Sassi et al., 2016). Primers for RT-qPCR are listed in Supplementary table 2. Gene expression levels were standardized using the expression level of the actin gene as the reference ( $\triangle \mathrm{CT}$ method). For genes GUT1, GUT2, $T P I 1$, and TKL1, the fold difference in gene expression between the mutants and the JMY2900 strain were calculated as $2^{-\Delta \Delta C T}$ (Livak and Schmittgen, 2001). Samples were analyzed in duplicate.

\subsection{Statistical analysis}

Statistical signification of results was assessed by analysis of variance (ANOVA) followed by Dunnett's multiple comparison tests.
Table 2

Dynamics of erythritol production and glycerol consumption in different strains grown in EPF medium in shake flasks for eight days.

\begin{tabular}{llllll}
\hline Strain & $\begin{array}{l}\text { Overexpressed } \\
\text { genes }\end{array}$ & $\begin{array}{l}\text { Biomass } \\
\left(\mathrm{g}_{\mathrm{DCW}} / \mathrm{L}\right)\end{array}$ & $\begin{array}{l}\mathrm{q}_{\mathrm{ERY}}(\mathrm{g} / \\
\left.\mathrm{g}_{\mathrm{DCW}} \cdot \mathrm{h}\right)\end{array}$ & $\begin{array}{l}\mathrm{q}_{\mathrm{GLY}}(\mathrm{g} / \\
\left.\mathrm{g}_{\mathrm{DCW}} \cdot \mathrm{h}\right)\end{array}$ & $\mathrm{Y}_{\mathrm{P} / \mathrm{S}}(\mathrm{g} / \mathrm{g})$ \\
\hline $\begin{array}{r}\text { JMY2900 } \\
\text { (WT) }\end{array}$ & - & 5.30 & 0.035 & 0.076 & 0.46 \\
FCY205 & GUT1 & 4.83 & $0.051^{*}$ & $0.091^{*}$ & $0.56^{*}$ \\
FCY206 & GUT2 & 4.83 & 0.038 & 0.068 & 0.56 \\
FCY207 & TPI1 & 5.02 & 0.039 & 0.071 & 0.54 \\
FCY208 & TKL1 & 5.36 & 0.040 & 0.068 & $0.59^{*}$ \\
FCY209 & YidA & 4.96 & 0.031 & 0.066 & 0.44 \\
FCY210 & ALR & $6.24^{*}$ & 0.028 & $0.049^{*}$ & $0.57^{*}$ \\
FCY213 & GUT1-GUT2 & 3.62 & $0.056^{*}$ & $0.102^{*}$ & 0.54 \\
FCY214 & GUT1-TKL1 & 4.81 & $0.058^{*}$ & $0.095^{*}$ & $0.61^{*}$ \\
FCY215 & GUT1-ALR & 4.48 & $0.058^{*}$ & $0.094^{*}$ & $0.61^{*}$ \\
FCY216 & GUT1-TPI1 & 4.65 & $0.048^{*}$ & 0.085 & $0.56^{*}$ \\
\hline
\end{tabular}

The values provided are the means of three independent replicates; the standard deviations represented less than $10 \%$ of the means. The abbreviations are as follows: $\mathrm{q}_{\mathrm{ERY}}$ : specific erythritol production rate; $\mathrm{q}_{\mathrm{GLY}}$ : specific glycerol consumption rate; and $\mathrm{Y}_{\mathrm{P} /}$ s: erythritol/glycerol conversion yield.

* Significantly different from JMY2900.

Alpha value was set at 0.05 . All statistical tests were performed using GraphPad Prism 6.0.2 software.

\section{Results and discussion}

\subsection{Overexpression of glycerol kinase increases glycerol consumption rate and erythritol productivity}

With the goal of enhancing erythritol productivity, we first attempted to improve glycerol consumption by overexpressing the genes GUT1 (which codes for GK; YALIOF00484g) and GUT2 (which codes for GDH; YALIOB13970g) either separately or simultaneously in the Y. lipolytica strain Po1d (Fig. 1 and Supplementary table 1). For strain FCY205 (pTEF-GUT1), the specific glycerol consumption rate ( $\mathrm{q}_{\mathrm{GLY}}$ ) was $20 \%$ higher than that of the wild type strain JMY2900 ( 0.091 and $0.076 \mathrm{~g} / \mathrm{g}_{\mathrm{DCW}} . \mathrm{h}$ respectively; Table 2 ). This increase is similar to that obtained for the Y. lipolytica A101 mutant that overexpressed GUT1 (Mirończuk et al., 2016). In contrast, strain FCY206 (pTEF-GUT2) showed a slightly lower glycerol consumption rate as compared to the wild type strain (Fig. 1) even though GUT2 expression was six times greater in FCY206 than in the wild type (Supplementary Fig. 3). Furthermore, strain FCY213, which overexpressed both GUT1 and GUT2, demonstrated just a slight increase in specific glycerol consumption rate compared to strain FCY205 (pTEF-GUT1) (0.102 and $0.091 \mathrm{~g} / \mathrm{g}_{\mathrm{DCW}} \cdot \mathrm{h}$, respectively). In $Y$. lipolytica strain A101, an $11 \%$ increase in glycerol consumption was observed when both GUT1 and GUT2 were coexpressed as compared to when just GUT1 was overexpressed (Mirończuk et al., 2016). For strains FCY205 (pTEF-GUT1) and FCY213 (pTEF-GUT1-GUT2), a similar increase of $\mathrm{q}_{\mathrm{GLY}}$ was observed (12\%; Table 2). Therefore, the simultaneous overexpression of GUT1 and GUT2 seems to have a combined effect on glycerol consumption under our experimental conditions. However, strain FCY213 (pTEF-GUT1-GUT2) had a 30\% lower maximum biomass as compared to the wild type (3.62 and $5.3 \mathrm{~g}_{\mathrm{DCW}} / \mathrm{L}$, respectively; Table 2).

In FCY205 (pTEF-GUT1), the specific erythritol production rate ( $\mathrm{q}_{\mathrm{ERY}}$ ) was $45 \%$ greater than in the wild type $(0.051$ and $0.035 \mathrm{~g} /$ $\mathrm{g}_{\mathrm{DCW}} \cdot \mathrm{h}$, respectively), while the glycerol/erythritol conversion yield $\left(\mathrm{Y}_{\mathrm{P} / \mathrm{S}}\right.$ ) was $21 \%$ higher ( 0.56 and $0.46 \mathrm{~g} / \mathrm{g}$, respectively). Surprisingly, and in contrast to the findings of Mirończuk et al. (2016), the overexpression of GUT2 (strain FCY206) had no effect on specific erythritol production rate $\left(0.038 \mathrm{~g} / \mathrm{g}_{\mathrm{DCw}} \cdot \mathrm{h}\right)$. In strain FCY213 (pTEFGUT1-GUT2), there was only a slight increase (9\%) in the specific erythritol production rate as compared to that in strain FCY205 (pTEFGUT1) ( 0.056 and $0.051 \mathrm{~g} / \mathrm{g}_{\mathrm{DCw}} \cdot \mathrm{h}$, respectively). Since the overexpres- 
sion of GUT1 led to an increase in both the specific glycerol consumption rate and the specific erythritol production rate, an additional strain, FCY212, was constructed that contained two copies of the pTEFGUT1 expression cassette. However, it did not display further improvement in erythritol productivity (data not shown).

\subsection{Overexpression of triose isomerase and transketolase leads to an increase in erythritol productivity}

Genes such as TPI1, TKL1, E4PP, and ER encode key enzymes involved in the pathway by which erythritol is synthesized from DHAP, the end product of glycerol catabolism (Fig. 1). They were overexpressed separately in the Po1d strain to assess their effects on erythritol productivity. Genes encoding TIM (TPI1; YALIOF05214g) and TK (TKL1; YALIOE06479g) were identified in the $Y$. lipolytica genome and used to construct strains FCY207 and FCY208, respectively. Unfortunately, erythrose-4P-phosphatase (E4PP) has yet to be characterized in yeast. However, Kuznetsova et al. (2006) reported that, in $E$. coli, a member of the haloacid dehalogenase-like hydrolase superfamily-HAD13 - showed a high degree of phosphatase activity directed toward erythrose-4-phosphate $\left(\mathrm{K}_{\mathrm{cat}} / \mathrm{K}_{\mathrm{m}}\right.$ value of $\left.10^{6}\right)$. Therefore, the corresponding YidA gene (EG11195) was cloned, assessed for codon compatibility in $Y$. lipolytica, and used to construct strain FCY209. We were also interested in ER. Past research has suggested that gene JX885666 in Y. lipolytica strain DSMZ70562 encodes an ER (Ghezelbash et al., 2014). However, a BlastN search using the gene sequence as a query did not find a corresponding gene in the genome of strain Po1d. Moreover, our attempts to amplify that particular gene using the primers designed by Ghezelbash and colleagues (ER1 and ER2; Supplementary table 2) were unsuccessful for both Po1d and DSMZ70562 (data not shown). In contrast, we did find a corresponding gene (FJ550210) in C. magnolia JH110 (Lee et al., 2010) using a BlastN search. Consequently, it was amplified from the genomic DNA of $C$. magnolia CBS2800 and used to construct strain FCY210.

These engineered strains were grown in EPF medium for eight days (i.e., until glycerol near-exhaustion). Strains FCY207 (pTEF-TPI1) and FCY208 (pTEF-TKL1) showed increased erythritol production compared to the wild type (5\% and $16 \%$, respectively; Fig. 2 and Supplementary Fig. 3). Strain FCY208 (pTEF-TKL1) had a higher conversion yield than did strain FCY205 (pTEF-GUT1) (0.59 and $0.56 \mathrm{~g} / \mathrm{g}$, respectively; Table 2). However, glycerol consumption was somewhat lower in the former $\left(0.068 \mathrm{~g} / \mathrm{g}_{\mathrm{DCW}} \cdot \mathrm{h}\right)$ than in the wild type strain $\left(0.076 \mathrm{~g} / \mathrm{g}_{\mathrm{DCW}} . \mathrm{h}\right)$. Strain FCY210, which overexpressed the $A L R$ gene taken from $C$. magnolia, did not have increased erythritol production (Fig. 2). To circumvent any potential problems that may have

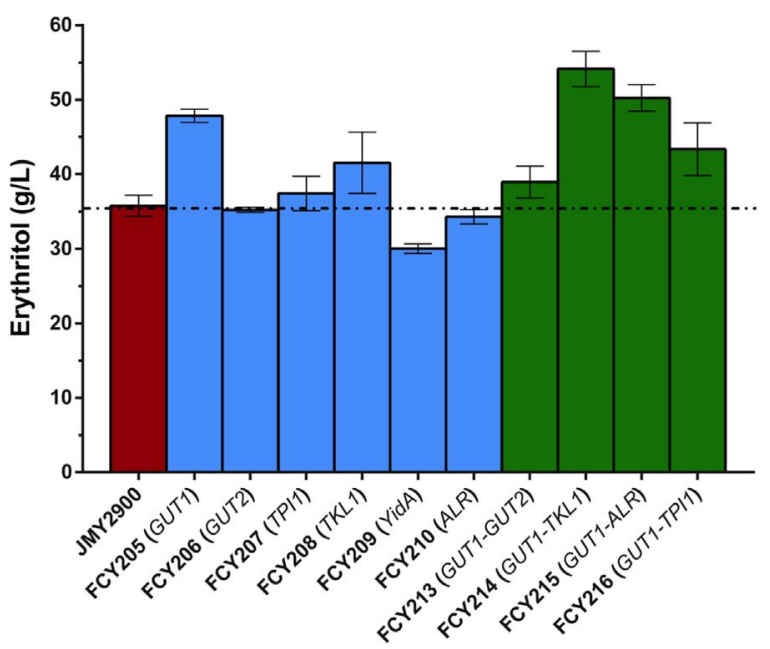

Fig. 2. Erythritol production of the study strains grown in EPF medium in shake flasks for eight days. occurred in the translation of ALR mRNA in strain FCY210, a codonoptimized version of $A L R$ ( $A L R \_C O$ ) was designed and used to construct strain FCY211. The latter did not show significantly improved erythritol productivity relative to strain FCY210 (data not shown). Strain FCY209, which over expressed E4PP (YidA), had a significantly lower level of erythritol production than the wild type (30 and $35 \mathrm{~g} / \mathrm{L}$, respectively) and a slower specific erythritol production rate $(0.031$ and $0.035 \mathrm{~g} /$ $\mathrm{g}_{\mathrm{DCW}} \cdot \mathrm{h}$, respectively). This negative effect of YidA overexpression could be linked to the ability of the YidA gene product to hydrolyze DHAP, as suggested by Kuznetsova et al. (2006).

\subsection{The pull and push strategy to enhance erythritol production}

As mentioned above, strain FCY205 (pTEF-GUT1) showed a significant increase in glycerol uptake, while strain FCY208 (pTEF-TKL1) displayed the highest conversion yield. Hence, to further increase erythritol productivity, GUT1 and TKL1 were coexpressed in strain FCY214 (pTEF-GUT1-TKL1I; Supplementary Fig. 3). In shake-flask cultures, this strain performed significantly better than the wild type, displaying a $65 \%$ increase in erythritol productivity. It combined the higher glycerol uptake capacity of strains FCY205 (pTEF-GUT1, 0.095 and $0.091 \mathrm{~g} / \mathrm{g}_{\mathrm{DC} W} \cdot \mathrm{h}$, respectively) and the higher conversion yields of FCY208 (pTEF-TKL1, 0.61 and $0.59 \mathrm{~g} / \mathrm{g}$, respectively). To further expand this push and pull strategy, strains FCY215 and FCY216 were constructed; they coexpressed GUT1-ALR and GUT1-TPI1, respectively. Although these two strains performed better than wild type, their erythritol production was not significantly greater than that of strain FCY214 (pTEF-GUT1-TKL1) (Fig. 2, Table 2). This suggests that glycerol assimilation and the redirection of glyceraldehyde-3-phosphate towards the non-oxidative part of pentose phosphate pathway are the main limiting steps for the synthesis of erythritol. Therefore, increasing the rate of these reactions could contribute to increasing the erythritol productivity and yield.

Next, we investigated the behavior of strain FCY214 (pTEF-GUT1TKL1) and the wild type under bioreactor conditions. The strains were cultured for $96 \mathrm{~h}$ in EPD medium, and cell growth, glycerol consumption, and erythritol production were monitored (Fig. 3). For strain FCY214 (pTEF-GUT1-TKL1), the final erythritol concentration in the culture supernatant was $79.4 \mathrm{~g} / \mathrm{L}$ (Table 3). This value was $42 \%$ greater than that obtained for the wild type $(55.8 \mathrm{~g} / \mathrm{L}$; Table 3$)$. Under bioreactor conditions, erythritol was produced at a high, constant rate ( $0.84 \mathrm{~g} / \mathrm{L} . \mathrm{h}$ ) between $24 \mathrm{~h}$ and the end of culture (Table 3 and Fig. 3). This result contrasts with those obtained for $Y$. lipolytica strain AJD pADUTGut1/2 (constitutive GUT1/GUT2 coexpression), whose erythritol production differed from that of the wild type (strain A101) only during the four last hours of culture (Mirończuk et al., 2016). Here, erythritol specific production rate was similar for the bioreactor and shake-flask cultures of strain FCY214 $\left(0.057\right.$ and $0.058 \mathrm{~g} / \mathrm{g}_{\mathrm{DCW}} \cdot \mathrm{h}$, respectively), while glycerol uptake was slightly higher in the bior-

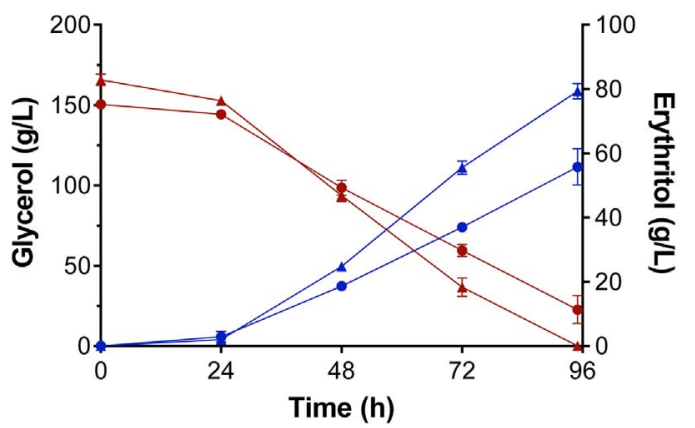

Fig. 3. Glycerol uptake (red line) and erythritol production (blue line) for FCY214 (pTEFGUT1-TKL1 triangles) and JMY2900 (WT; circles) grown in EPB medium in a bioreactor. (For interpretation of the references to color in this figure legend, the reader is referred to the web version of this article). 
Table 3

Dynamics of erythritol production and glycerol consumption for bioreactor cultures of JMY2900 (WT), FCY214, and FCY218. The standard deviations represented less than 10\% of the means. The abbreviations are the same as in Table 2 .

\begin{tabular}{llll}
\hline & JMY2900 & FCY214 & FCY218 \\
\hline Erythritol production (g/L) & 55.8 & 79.4 & 80.6 \\
Erythritol productivity (g/L.h) & 0.59 & 0.84 & 1.03 \\
$\mathrm{q}_{\mathrm{ERY}}\left(\mathrm{g} / \mathrm{g}_{\mathrm{DCW}} \cdot \mathrm{h}\right)$ & 0.046 & 0.057 & 0.073 \\
$\mathrm{q}_{\mathrm{GLY}}\left(\mathrm{g} / \mathrm{g}_{\mathrm{DCW}} \cdot \mathrm{h}\right)$ & 0.105 & 0.119 & 0.138 \\
$\mathrm{Y}_{\mathrm{P} / \mathrm{S}}(\mathrm{g} / \mathrm{g})$ & 0.44 & 0.48 & 0.53 \\
Final biomass $\left(\mathrm{g}_{\mathrm{DCW}} / \mathrm{L}\right)$ & 12.8 & 14.7 & 14.6 \\
\hline
\end{tabular}

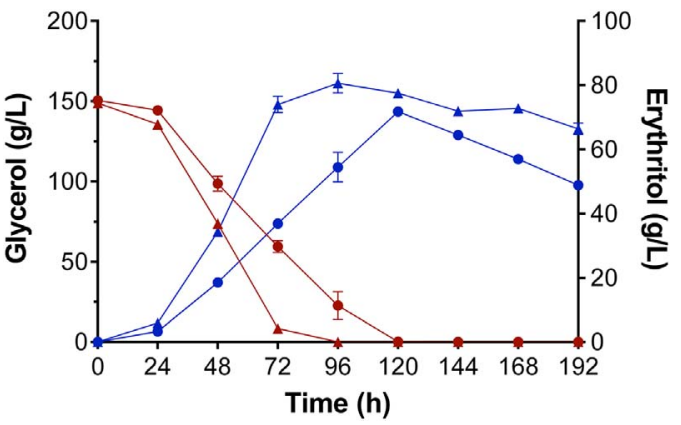

Fig. 4. Glycerol uptake (red line) and erythritol production (blue line) for FCY218 (pTEFGUT1-TKL1- $\Delta$ eyk1; triangles) and JMY2900 (WT; circles) grown in EPB medium in a bioreactor. (For interpretation of the references to color in this figure legend, the reader is referred to the web version of this article).

eactor (0.119 vs. $\left.0.095 \mathrm{~g} / \mathrm{g}_{\mathrm{DCW}} \cdot \mathrm{h}\right)$.

\subsection{Disruption of EYK1 in strain FCY214 further increases erythritol productivity}

The ability of $Y$. lipolytica to catabolize erythritol alongside glycerol negatively affects erythritol productivity and conversion yield. Recently, we discovered that the gene EYK1 (YALIOF01606g) is involved in erythritol catabolism (Carly et al., submitted for publication). Therefore, this gene was disrupted in a strain overexpressing GUT1 and TKL1 (Supplementary Fig. 2). As expected, the resulting strain, FCY218, was unable to consume erythritol, especially after the glycerol source had been exhausted in the culture medium (Fig. 4). In those conditions, erythrulose, the byproduct of erythritol oxidation, started to accumulate in the culture medium after glycerol depletion, however in small amount (less than $3 \%$ of the produced erythritol was converted after $24 \mathrm{~h}$ of glycerol depletion). Consequently, strain FCY218 performed better than strain FCY214 (glycerol specific consumption rate: $0.138 \mathrm{vs.} 0.119 \mathrm{~g} / \mathrm{g}_{\mathrm{DCw}} \cdot \mathrm{h}$; erythritol specific production rate: 1.03 vs. $0.84 \mathrm{~g} / \mathrm{L} . \mathrm{h}$; and conversion yield: 0.53 vs. $0.48 \mathrm{~g} / \mathrm{g}$; Table 3). Moreover, the maximum concentration of erythritol was obtained in $40 \%$ less time than in the wild type strain, which further emphasizes the potential benefits of this system.

\section{Conclusion}

In the past, erythritol productivity in $Y$. lipolytica has largely been improved by classical approaches that consisted of optimizing either the culture medium or culturing conditions (Rymowicz et al., 2008, Mirończuk et al., 2014, Yang et al., 2014). Recently, however, Mirończuk et al. (2016) reported that metabolic engineering could increase the rate of glycerol catabolism, resulting in a 35\% increase in erythritol productivity. In this study, we attempted to make further progress by increasing the flow of carbon through the erythritol synthesis pathway, notably in the transitions from erythrose phosphate to erythritol. Our approach resulted in a $65 \%$ increase in erythritol specific production rate relative to the wild type. A major challenge in the development of an efficient process for producing erythritol is the ability of $Y$. lipolytica to catabolize erythritol alongside glycerol. Here, we also constructed a strain whose erythritol catabolism was impaired. In this mutant, erythritol productivity was increased $78 \%$ relative to the wild type and maximum concentrations were obtained in $40 \%$ less time. Moreover, we achieved these values using an inexpensive medium and without having optimized culturing conditions. The next step is to develop a glycerol fed-batch fermentation method that can further increase the specific erythritol production rate as well as yield.

\section{Funding}

F. Carly and M. Vandermies each received a fellowship from the Belgian Fund for Research Training in Industry and Agriculture (FRIA), Grant No. (FC00572).

\section{Conflict of interest}

The authors declare that they have no conflicts of interest.

\section{Appendix A. Supporting information}

Supplementary data associated with this article can be found in the online version at http://dx.doi.org/10.1016/j.ymben.2017.05.002.

\section{References}

Almeida, J.R.M., Fávaro, L.C.L., Quirino, B.F., 2012. Biodiesel biorefinery: opportunities and challenges for microbial production of fuels and chemicals from glycerol waste. Biotechnol. Biofuels 5, 48.

Bernt, W.O., Borzelleca, J.F., Flamm, G., Munro, I.C., 1996. Erythritol: a review of biological and toxicological studies. Regul. Toxicol. Pharmacol. 24, S191-S197.

Barth, G., Gaillardin, C., 1996. Yarrowia lipolytica. In Nonconventional Yeasts in Biotechnology. Springer Berlin Heidelberg, Berlin, Heidelberg, pp. 313-388.

Carly, F., Gamboa-Melendez, H., Vandermies, M., Damblon, C., Nicaud, J.-M., Fickers, P., 2017. Identification and characterization of EYK1, a key gene for erythritol catabolism in Yarrowia lipolytica. Appl. Microbiol. Biotechnol (submitted for publication).

Fickers, P., Benetti, P., Wache, Y., Marty, A., Mauersberger, S., Smit, M., Nicaud, J.-M., 2005. Hydrophobic substrate utilisation by the yeast Yarrowia lipolytica, and its potential applications. FEMS Yeast Res. 5, 527-543.

Ghezelbash, G.R., Nahvi, I., Malekpour, A., 2014. Erythritol production with minimum by-product using Candida magnoliae mutant. Appl. Biochem. Microbiol. 50, 292-296.

Hallsworth, J.E., Magan, N., 1997. A rapid HPLC protocol for detection of polyols and trehalose. J. Microbiol. Methods 29, 7-13.

Higuchi, R., Krummel, B., Saiki, R.K., 1988. A general method of in vitro preparation and specific mutagenesis of DNA fragments: study of protein and DNA interactions. Nucleic Acids Res. 16, 7351-7367.

Ishizuka, H., Wako, K., Kasumi, T., Sasaki, T., 1989. Breeding of a mutant of Aureobasidium sp. with high erythritol production. J. Ferment. Bioeng. 68, 310-314.

Jeya, M., Lee, K.-M., Tiwari, M.K., Kim, J.-S., Gunasekaran, P., Kim, S.-Y., Kim, I.-W., Lee, J.-K., 2009. Isolation of a novel high erythritol-producing Pseudozyma tsukubaensis and scale-up of erythritol fermentation to industrial level. Appl. Microbiol. Biotechnol. 83, 225-231.

Kawanabe, J., Hirasawa, M., Takeuchi, T., Oda, T., Ikeda, T., 1992. Noncariogenicity of erythritol as a substrate. Caries Res. 26, 358-362.

Kim, S.-Y., Lee, K.-H., Kim, J.-H., Oh, D.-K., 1997. Erythritol production by controlling osmotic pressure in Trigonopsis variabilis. Biotechnol. Lett. 19, 727-729.

Kuznetsova, E., Proudfoot, M., Gonzalez, C.F., Brown, G., Omelchenko, M.V., Borozan, I., Carmel, L., Wolf, Y.I., Mori, H., Savchenko, A.V., et al., 2006. Genome-wide analysis of substrate specificities of the Escherichia coli haloacid dehalogenase-like phosphatase family. J. Biol. Chem. 281, 36149-36161.

Lee, J.-K., Ha, S.-J., Kim, S.-Y., Oh, D.-K., 2000. Increased erythritol production in Torula sp. by Mn2+ and Cu2 +. Biotechnol. Lett. 22, 983-986.

Lee, D.-H., Lee, Y.-J., Ryu, Y.-W., Seo, J.-H., 2010. Molecular cloning and biochemical characterization of a novel erythrose reductase from Candida magnoliae JH110. Microb. Cell Factor. 9, 43.

Lin, S.-J., Wen, C.-Y., Wang, P.-M., Huang, J.-C., Wei, C.-L., Chang, J.-W., Chu, W.-S., 2010. High-level production of erythritol by mutants of osmophilic Moniliella sp. Process Biochem. 45, 973-979.

Livak, K.J., Schmittgen, T.D., 2001. Analysis of relative gene expression data using realtime quantitative PCR and the $2-\Delta \Delta$ CT method. Methods 25, 402-408.

Mirończuk, A.M., Furgała, J., Rakicka, M., Rymowicz, W., 2014. Enhanced production of erythritol by Yarrowia lipolytica on glycerol in repeated batch cultures. J. Ind. Microbiol. Biotechnol. 41, 57-64.

Mirończuk, A.M., Rakicka, M., Biegalska, A., Rymowicz, W., Dobrowolski, A., 2015. A two-stage fermentation process of erythritol production by yeast $Y$. lipolytica from 
molasses and glycerol. Bioresour. Technol. 198, 445-455.

Mirończuk, A.M., Rzechonek, D.A., Biegalska, A., Rakicka, M., Dobrowolski, A., 2016. A novel strain of Yarrowia lipolytica as a platform for value-added product synthesis from glycerol. Biotechnol. Biofuels 9, 180-191.

Moon, H.-J., Jeya, M., Kim, I.-W., Lee, J.-K., 2010. Biotechnological production of erythritol and its applications. Appl. Microbiol. Biotechnol. 86, 1017-1025.

Munro, I., Bernt, W., Borzelleca, J., Flamm, G., Lynch, B., Kennepohl, E., Bär, E. Modderman, J., 1998. Erythritol: an interpretive summary of biochemical, metabolic, toxicological and clinical data. Food Chem. Toxicol. 36, 1139-1174.

Nicaud, J.-M., 2012. Yarrowia lipolytica. Yeast 29, 409-418.

Paradowska, K., Nitka, D., 2009. Purification and characterization of erythrito dehydrogenase from Mycobacterium smegmatis. Ann. UMCS Pharm. 22, 47-55.

Park, Y.-C., Oh, E.J., Jo, J.-H., Jin, Y.-S., Seo, J.-H., 2016. Recent advances in biological production of sugar alcohols. Curr. Opin. Biotechnol. 37, 105-113.

Querol, A., Barrio, E., Huerta, T., Ramón, D., 1992. Molecular monitoring of wine fermentations conducted by active dry yeast strains. Appl. Environ. Microbiol. 58, 2948-2953.

Rakicka, M., Rywińska, A., Cybulski, K., Rymowicz, W., 2016. Enhanced production of erythritol and mannitol by Yarrowia lipolytica in media containing surfactants. Braz. J. Microbiol. 47, 417-423.

Rymowicz, W., Rywińska, A., Gładkowski, W., 2008. Simultaneous production of citric acid and erythritol from crude glycerol by Yarrowia lipolytica Wratislavia K1. Chem. Pap. 62, 239-246.

Ryu, Y.-W., Park, C.Y., Park, J.B., Kim, S.-Y., Seo, J.-H., 2000. Optimization of erythritol production by Candida magnoliae in fed-batch culture. J. Ind. Microbiol. Biotechnol. 25, 100-103.
Rywińska, A., Juszczyk, P., Wojtatowicz, M., Robak, M., Lazar, Z., Tomaszewska, L., Rymowicz, W., 2013. Glycerol as a promising substrate for Yarrowia lipolytica biotechnological applications. Biomass-Bioenergy 48, 148-166.

Sambrook, J., Fritsch, E., Maniatis, T., 1989. Molecular Cloning: A Laboratory Manual: Vol. 2 (S.l.: Cold Spring Harbor).

Sassi, H., Delvigne, F., Kar, T., Nicaud, J.-M., Coq, A.-M.C.-L., Steels, S., Fickers, P., 2016 Deciphering how LIP2 and POX2 promoters can optimally regulate recombinant protein production in the yeast Yarrowia lipolytica. Microb. Cell Factor. 15, 159-169.

Sawada, K., Taki, A., Yamakawa, T., Seki, M., 2009. Key role for transketolase activity in erythritol production by Trichosporonoides megachiliensis SN-G42. J. Biosci. Bioeng. 108, 385-390.

Tomaszewska, L., Rywińska, A., Gładkowski, W., 2012. Production of erythritol and mannitol by Yarrowia lipolytica yeast in media containing glycerol. J. Ind. Microbiol. Biotechnol. 39, 1333-1343.

Vandermies, M., Denies, O., Nicaud, J.M., Fickers, P., 2017. EYK encoding erythrulose kinase as a catabolic selectable marker for genome editing in the non-conventional yeast Yarrowia lipolytica. J. Microb. Methods (in press).

Yang, L.-B., Dai, X.-M., Zheng, Z.-Y., Zhu, L., Zhan, X.-B., Lin, C.-C., 2015. Proteomic analysis of erythritol-producing Yarrowia lipolytica from glycerol in response to osmotic pressure. J. Microbiol. Biotechnol. 7, 1059-1069.

Yang, L.-B., Zhan, X.-B., Zheng, Z.-Y., Wu, J.-R., Gao, M.-J., Lin, C.-C., 2014. A novel osmotic pressure control fed-batch fermentation strategy for improvement of erythritol production by Yarrowia lipolytica from glycerol. Bioresour. Technol. 151, $120-127$.

Zinjarde, J., 2014. Food-related application of Yarrowia lipolytica. Food Chem. 152, 1-10. 